\title{
Relativistic Corrections for Calculating Ionization Energies of One- to Five-Electron Isoelectronic Atomic Ions
}

\author{
Peter F. Lang and Barry C. Smith \\ School of Biological and Chemical Sciences, Birkbeck College, University of London, Malet Street, London WC1E 7HX, UK \\ Correspondence should be addressed to Peter F. Lang; p.f.lang@gmail.com
}

Received 20 August 2012; Accepted 1 October 2012

Academic Editors: A. M. Fonseca, A. Karadag, and Z. Wu

Copyright (C) 2013 P. F. Lang and B. C. Smith. This is an open access article distributed under the Creative Commons Attribution License, which permits unrestricted use, distribution, and reproduction in any medium, provided the original work is properly cited.

\begin{abstract}
We have previously proposed a simple empirical equation to reproduce the literature values of the ionization energies of oneelectron to four-electron atomic ions with very good agreement. However, we used a potential energy approach in our equation, which has no theoretical basis. This paper discusses an alternative kinetic energy expression for one to five electrons with simple corrections for relativistic and Lamb shift effects and for two- to-five electron ions additional effects including electron relaxation and residual interactions. For calculated values of one-electron (hydrogen-like) and two electron (helium like) atomic ions, the difference with the literature values is typically $0.001 \%$ or less. Agreement with the literature values for three-, four-, and fiveelectron ions is $99 \%$ or better. First electron affinities calculated by our expression also agree fairly well with generally recommended values. These results show that there is strong evidence that our methodology can be developed to reliably predict, with fairly good accuracy, ionization energies of multielectron atomic ions that have not been measured.
\end{abstract}

\section{Introduction}

A knowledge of ionization energies is essential for understanding the chemistry of the elements and other fundamental concepts, such as lattice energies of inorganic solids.

With the development of quantum theory, the twoparticle problem can be solved exactly and the kinetic energy of the electron in a hydrogen atom can be calculated using the Schrödinger equation. Since the Schrödinger equation does not take account of relativistic effects, Dirac [1] produced an equation which included a relativistic correction for the electron energy levels. However, Lamb and Retherford [2-4] in a series of experiments showed that there is a small shift in the energy levels of the hydrogen atom not accounted for by the Dirac equation. This energy shift is now commonly called the Lamb shift. Theoretical atomic energy levels were calculated from a nonrelativistic model, and then relativistic and quantum electrodynamic effects were accounted for by treating them as perturbation corrections.

General availability of powerful computers allowed highly complicated theoretical calculations of the energy levels and ionization energies of hydrogen [5] and heliumlike ions [6] to be performed. These sophisticated equations for one- and two-electron atomic ions, which need complex computer routines to compute, include corrections for the variation of mass with velocity, reduced mass, mass polarization, and Lamb shift, and for two-electron ions, corrections for interactions between the two electrons. Values computed by these equations are quoted to $\mathrm{cm}^{-1}$ (wave number) level or small fractions of $\mathrm{a} \mathrm{cm}^{-1}$. Updated theoretical ionization energies and Lamb shifts for one-electron [7] and twoelectron [8] atomic ions have been published more recently and are generally accepted as very accurate. These recent results differ very little from the earlier computed values of Garcia and Mack [5] or Midtdal and Aashamar [6].

We have previously devised a simple empirical equation to reproduce literature values for the ionization energies of one-electron [9] and two-electron [10] atomic ions with very good agreement. More recently, we extended calculations to three- and four-electron isoelectronic ions [11], which reproduced literature values with good agreement. However, this equation is based on a potential energy approach (when the 
potential energy is maximum, the kinetic energy approaches zero, that is, zero velocity, and relativistic correction for the variation of mass with velocity is not required) and has no theoretical basis. In this paper we use a kinetic energy expression with a relativistic correction, which has theoretical justification to calculate the ionization energies of one- to five-electron atomic ions. Besides applying a relativistic and a Lamb shift correction, we have also made a few hypotheses on factors that influence the size of the ionization energy. To maintain our aim of simplicity and ease of computation, the equations only contain fundamental constants or values derived from fundamental constants and simple numbers.

\section{Sources of Data}

The extensive publications by Moore [12-15] contain detailed tables of atomic energy levels in reciprocal wavelengths or wave numbers $\left(\mathrm{cm}^{-1}\right)$, ionization reciprocal wavelengths (in $\mathrm{cm}^{-1}$ ), and values converted from wave numbers to electron volts $(\mathrm{eV})$ for atoms and atomic ions with estimated experimental errors and references to original work. This remains the most extensive survey of ionization energies. Recently, the CRC Handbook of Chemistry and Physics [16] presented extensive authoritative data of ionization energies with reference to Moore and other up-to-date works. Andersen et al. [17] provided an extensive compilation of first electron affinities (binding energies of single negative ions) based on both theory and experiment. The majority of compilations of ionization energy data are now available on the National Institute of Standards and Technology web site (http://www.nist.gov/srd/). These compilations include values of ionization energies that are accurately measured as well as crude estimates.

On closer examination of many of the original papers and compilations, we have found that, in general, the first few ionization energies are most reliably measured, experimental errors increase across the higher members of a series, and some results have errors of over one eV. For example, the ionization energy of $\mathrm{Sc}_{\mathrm{XVII}}$ (five-electron boron series) is $1094 \pm 2 \mathrm{eV}^{18}$. Hence, we consider that when comparisons are made, agreement with the first few members of a series is more relevant than with higher members of a series. There is no reason to doubt the reliability of the values published in the CRC Handbook of Chemistry and Physics of all fundamental constants and ionization energies. Values of all fundamental constants used for calculation in this work and ionization energies for comparison purposes are taken directly from it. Table 1 shows the symbols, elementary constants and conversion factors used in this work.

Ionization energies are presented in electron volts $(\mathrm{eV})$ in the Handbook of Chemistry and Physics and $\mathrm{eV}$ is a more commonly used unit, so, as with our previous work, ionization energies are presented here in $\mathrm{eV}$ and rounded to four decimal places (please note that $0.0001 \mathrm{eV}$ is equivalent to $0.8 \mathrm{~cm}^{-1}$.) We consider that this is sufficiently precise, since some literature values are quoted only to one or two decimal places or less because the levels of uncertainty/experimental error for some ionization energies can be of the order of an $\mathrm{eV}$
TABLE 1: Symbols, conversion factors, and constants (to 9 significant figures).

\begin{tabular}{ll}
\hline Symbol/constant & Value/comment/definition \\
\hline$\alpha$ & 0.00729735254 \\
$I$ & $\begin{array}{l}\text { Number of electrons remaining after } \\
\text { ionization }\end{array}$ \\
$k$ & $01.23984180 \times 10^{-4}$ \\
$n$ & Principal quantum number \\
$v$ & Electron velocity \\
$Q$ & Number of residual electron-electron \\
$A$ & interactions \\
$\mu$ & Mass number \\
$S$ & Reduced mass (see Table 8 column 6 for \\
$S_{1}$ & individual values) \\
$S_{2}$ & Screening constant for the ionizing electron \\
$Z$ & Screening for the remaining electron $(\mathrm{s})$ after \\
$c$ & ionization \\
$m_{\mathrm{e}}$ & Screening for the remaining electron $(\mathrm{s})$ \\
$m_{p}$ & before ionization \\
$q$ & Proton number (nuclear charge) \\
$h$ & Velocity of light $2.99792458 \times 10^{8} \mathrm{~ms}{ }^{-1}$ \\
$\varepsilon_{0}$ & Electron rest mass $9.10938215 \times 10^{-31} \mathrm{~kg}$ \\
\hline & Proton rest mass $1.67262164 \times 10^{-27} \mathrm{~kg}$ \\
& Electron charge $1.60217649 \times 10^{-19} \mathrm{C}$ \\
& Planck's constant $6.62606896 \times 10^{-34} \mathrm{Js}$ \\
& $8.85418782 \times 10^{-12} \mathrm{Fm}^{-1}$ \\
&
\end{tabular}

or more. For comparison purposes, values that are originally presented in $\mathrm{cm}^{-1}$ are converted to $\mathrm{eV}$ with the conversion factor $k$ in Table 1. Results are presented up to atomic number 20 for the following two reasons. Firstly, uncertainties of the ionization energies of those elements with atomic number 21 or more (for an isoelectronic sequence) are usually much higher than the differences between the calculated values produced by our expression and literature values for elements with atomic number below 21. Secondly, our results are compared with ionization energies computed by Garcia and Mack [5] and Midtdal and Aashamar [6] who presented their results up to atomic number 20.

\section{Contributions to the Electron Energy}

The kinetic energy of an electron in an isolated atom or atomic ion is not simply $(1 / 2) m_{0} v^{2}$ because it is moving at high speed. When there is more than one electron in the system, it is logical to assume that the attractive energy between the nucleus and remaining electron(s) changes because of the change in screening experienced by the remaining electron(s) before and after ionization. The energy released by the electron relaxation or transition needs to be taken into account as well as the Lamb shift correction when calculating the ionization energy. There may be residual electron interactions because of the finite size of the electron. Hence, we assume that the ionization energy of an electron 
can be considered to contain the following components in a multielectron system:

$$
E_{i}=\frac{1}{n^{2}} \mu\left(J_{k}-J_{l}-J_{t}\right) \pm \mu E_{r}=E_{k}-E_{l}-E_{t} \pm \mu E_{r},
$$

where $E_{i}$ is the ionization energy, $J_{k}$ is the kinetic energy term, $J_{l}$ is the Lamb shift term, $J_{t}$ is the relaxation/transition energy term, and $E_{r}$ represents residual electron interaction energy term. $n$ is the principal quantum number and $\mu$ is the reduced mass [18] (because the nucleus and electron being ionized, both revolve around the centre of mass). $E_{k}, E_{l}$, and $E_{t}$ represent $J_{k}, J_{l}, J_{t}$ multiplied by $1 / n^{2}$, respectively, because ionization occurs in energy level $n$, where $n$ is the principal quantum number. Values of ionization energy calculated by our expression are converted to $\mathrm{eV}$ by dividing them with the elementary electron charge (given in Table 1).

\section{Kinetic Energy and the Relativistic Correction}

The maximum velocity of the electron moving in a Bohr orbit [19] can be calculated from the relationship:

$$
\frac{m_{0} v_{0}^{2}}{a_{0}}=\frac{q_{1} q_{2}}{\left(4 \pi \varepsilon_{0} a_{0}^{2}\right)}
$$

where $m_{0}$ is the electron rest mass, $v_{0}$ is the velocity of the electron, $q_{1} q_{2}$ stand for the charges of the electron and nucleus, $\varepsilon_{0}$ is the permittivity of a vacuum, and $a_{0}$, the Bohr radius, is $\varepsilon_{0} h^{2} / \pi m_{e} c^{2}$. The velocity $v_{0}$ of the electron in the hydrogen atom can be calculated from (2) and is equal to $2.18769125 \times 10^{6} \mathrm{~m} / \mathrm{sec}$. The velocity $v$ of the electron in successive atoms of the one-electron series increase by $Z$ times, where $Z$ is the atomic/proton number or $v=$ $v_{0} Z$. When there is more than one electron in the system, the velocity of the electron increases by $(Z-S)$, where $S$ is the screening constant for that electron. A common interpretation of relativity theory [20] shows the total energy of a particle of mass $m_{0}$ and velocity $v$ is

$$
E=m_{0} c^{2}+\frac{1}{2} m_{0} v^{2}+\frac{(3 / 8) m_{0} v^{4}}{c^{2}}+\frac{(5 / 16) m_{0} v^{6}}{c^{4}}+\cdots
$$

and the kinetic energy of the particle is then

$$
E_{k}=\frac{1}{2} m_{0} v^{2}+\frac{(3 / 8) m_{0} v^{4}}{c^{2}}+\frac{(5 / 16) m_{0} v^{6}}{c^{4}}+\cdots
$$

In reality, only the first three terms (in (4)) are significant as shown in the expression, and all other terms are minute and can be ignored. In an equilibrium situation, the energy of the electron is exactly half potential and half kinetic. Assuming that an electron is ionized in an equilibrium condition, only half the relativistic correction should be included, that is, the relativistic correction for a one-electron atom is then

$$
0.5\left(\frac{(3 / 8) m_{0} v^{4}}{c^{2}}+\frac{(5 / 16) m_{0} v^{6}}{c^{4}}\right)
$$

For a hydrogen-like ion, there is no relaxation energy and no residual electron interactions, and the ionization energy is then represented by

$$
E=\mu\left(\frac{1}{2} m_{0} v^{2}+0.5\left(\frac{(3 / 8) m_{0} v^{4}}{c^{2}}+\frac{(5 / 16) m_{0} v^{6}}{c^{4}}\right)-E_{l}\right) .
$$

Columns 2, 3, 5, and 6 of Table 2 list the nonrelativistic kinetic energy, the relativistic correction calculated by (5), the Lamb shift, and the ionization energy as calculated by (6), respectively. However, as shown below, values calculated by (6) for one-electron atoms are too large when compared to generally accepted values.

We consider that, as theory of relativity points out, the mass $m$ of a moving particle is given by the expression $m_{0} /\left(\sqrt{\left(1-v^{2} / c^{2}\right)}\right)$, where $m_{0}$ is the rest mass of particle. Expansion of this expression gives

$$
m=m_{0}\left(1+\frac{(1 / 2) v^{2}}{c^{2}}+\frac{(3 / 8) v^{4}}{c^{4}}+\frac{(5 / 16) v^{6}}{c^{6}}+\cdots\right) .
$$

Therefore, it follows that $(1 / 2) m v^{2}=(1 / 2) m_{0} v^{2}(1+$ $\left.(1 / 2) v^{2} / c^{2}+(3 / 8) v^{4} / c^{4}+(5 / 16) v^{6} / c^{6}+\cdots\right)$.

This expression for total kinetic energy can be simplified to:

$$
\frac{1}{2} m_{0} v^{2}+\frac{(1 / 4) m_{0} v^{4}}{c^{2}}+\frac{(3 / 16) m_{0} v^{6}}{c^{4}}+\cdots,
$$

and the relativistic correction for a one electron atom at the equilibrium position is then

$$
0.5\left(\frac{(1 / 4) m_{0} v^{4}}{c^{2}}+\frac{(3 / 16) m_{0} v^{6}}{c^{4}}\right)
$$

The ionization energy of a one-electron atom is then

$$
I=\mu\left(\frac{1}{2} m_{0} v^{2}+0.5\left(\frac{(1 / 4) m_{0} v^{4}}{c^{2}}+\frac{(3 / 16) m_{0} v^{6}}{c^{4}}\right)-E_{l}\right) .
$$

Columns 4 and 7 of Table 2 list the relativistic corrections calculated by (9) and the ionization calculated by (10), respectively. As described below, when expression (9) is used, the ionization energies calculated have excellent agreement with values calculated by complex computer routines and other generally accepted literature values.

\section{The Lamb Shift}

The Lamb Shift is usually considered as a quantum electrodynamic effect (see e.g., Garcia and Mack [5] or Johnson and Soff [7]) and is computed by complex formulas.

We have made a number of assumptions (without theoretical justification) that the Lamb shift is a secondary relativistic effect. We assume that the Lamb shift is caused by a combination of effects related to mass and size and the whole 
TABLE 2: Kinetic energy with two different relativistic and Lamb shift corrections for ionization energies of one-electron atomic ions (eV).

\begin{tabular}{|c|c|c|c|c|c|c|}
\hline \multirow{2}{*}{$Z$} & (2) & (3) & (4) & (5) & (6) & (7) \\
\hline & $\mu(1 / 2) m_{\mathrm{o}} v^{2}$ & Corr (4) & Corr (7) & $E_{1}$ & $I_{1}(5)$ & $I_{1}(9)$ \\
\hline 1 & 13.5983 & 0.0003 & 0.0002 & 0.0000 & 13.5985 & 13.5984 \\
\hline 2 & 54.4153 & 0.0043 & 0.0029 & 0.0005 & 54.4192 & 54.4177 \\
\hline 3 & 122.4416 & 0.0220 & 0.0147 & 0.0022 & 122.4615 & 122.4541 \\
\hline 4 & 217.6778 & 0.0696 & 0.0464 & 0.0060 & 217.7414 & 217.7182 \\
\hline 5 & 340.1253 & 0.1700 & 0.1133 & 0.0131 & 340.2823 & 340.2256 \\
\hline 6 & 489.7825 & 0.3527 & 0.2351 & 0.0241 & 490.1111 & 489.9935 \\
\hline 7 & 666.6528 & 0.6537 & 0.4357 & 0.0415 & 667.2651 & 667.0470 \\
\hline 8 & 870.7344 & 1.1160 & 0.7438 & 0.0666 & 871.7838 & 871.4416 \\
\hline 9 & 1102.0292 & 1.7890 & 1.1922 & 0.1028 & 1103.7154 & 1103.1187 \\
\hline 10 & 1360.5319 & 2.7289 & 1.8185 & 0.1465 & 1363.1143 & 1362.2039 \\
\hline 11 & 1646.2494 & 3.9992 & 2.6647 & 0.2082 & 1650.0404 & 1648.7060 \\
\hline 12 & 1959.1748 & 5.6698 & 3.7774 & 0.2789 & 1964.5657 & 1962.6733 \\
\hline 13 & 2299.3152 & 7.8179 & 5.2081 & 0.3748 & 2306.7584 & 2304.1485 \\
\hline 14 & 2666.6634 & 10.5280 & 7.0126 & 0.4809 & 2676.7105 & 2673.1951 \\
\hline 15 & 3061.2265 & 13.8917 & 9.2520 & 0.6204 & 3074.4978 & 3069.8581 \\
\hline 16 & 3482.9974 & 18.0078 & 11.9917 & 0.7708 & 3500.2345 & 3494.2184 \\
\hline 17 & 3931.9834 & 22.9829 & 15.3026 & 0.9642 & 3954.0021 & 3946.3218 \\
\hline 18 & 4408.1838 & 28.9312 & 19.2601 & 1.2104 & 4435.9046 & 4426.2335 \\
\hline 19 & 4911.5858 & 35.9744 & 23.9451 & 1.4269 & 4946.1332 & 4934.1039 \\
\hline 20 & 5442.2023 & 44.2423 & 29.4434 & 1.6957 & 5484.7489 & 5469.9500 \\
\hline
\end{tabular}

system in motion. The energy reduction is approximated to the following expression:

$$
E_{l}=\frac{m_{e}}{\left(m_{e}+m_{p}\right)} \times\left[\left(\frac{\alpha}{2^{0.667}}\right)\left(\left(\frac{1}{2}\right) m_{0} v_{0}^{2}\right)(Z-S)^{3.2} A^{1 / 3}\right],
$$

where $m_{e}$ is the electron mass, $m_{p}$ is the proton mass, where the factor for the reduced mass correction for hydrogen is $m_{e} /\left(m_{e}+m_{p}\right) \cdot \alpha$ is the fine structure constant and $\left(\alpha / 2^{0.667}\right)$ is a crude approximation of the square root of the ratio of nuclear to atomic size for hydrogen. $A$ is the mass number of the atom. $S$ is the screening constant and in a one-electron system $S$ is zero. The size of the nucleus increases [21] roughly in proportion to $A^{1 / 3}$. As shown in Table 3, the Lamb shift values calculated using this simple equation agree well with those computed by Johnson and Soff [7] for the one-electron system. For the two-electron system, the values calculated by (11) are higher than those computed by Midtdal and Aashamar [6], but they compare well with those computed by Drake [8], which are more recent and probably more accurate.

\section{Electron Relaxation Energy}

After an electron is ionized, one or more of the remaining electron(s) is/are attracted more closely to the nucleus. The attractive energy between the nucleus and remaining electron(s) changes because of the change in screening experienced by the remaining electron(s) before and after ionization. This relaxation or transition energy is proportional to

$$
\frac{n^{2}}{4} \times\left[\left(\frac{1}{2}\right) m_{0}\left(v_{0}\left(Z-S_{1}\right)\right)^{2}-\left(\frac{1}{2}\right) m_{0}\left(v_{0}\left(Z-S_{2}\right)\right)^{2}\right],
$$

where $S_{1}$ is the screening constant for remaining electron(s) after ionization and $S_{2}$ is the screening constant for the remaining electron(s) before ionization.

In the helium system, there are two electrons and both occupy the 1s orbital. Since each electron occupies half of the space and each is repelled by only one other electron (here we have assumed that the two electrons act as in a two-particle problem and are equivalent), the screening constant is a half (0.5). After ionization, there is only one electron in the system and so there is zero screening.

In the lithium series, the electron that is ionized occupies a higher (2s) orbital and is shielded by two 1s electrons, and the screening increases by 1 to 1.5 . The extra screening experienced by the two inner electrons in the $1 \mathrm{~s}$ orbital increases to 0.625 , which is an increase by $1 / 8$ or 0.125 rather than 0.5 because the third electron occupies a different orbital and in a different electron shell (i.e., 0.5 of 0.5 of 0.5 ). After ionization, only two electrons are left in the system and the screening reduces to just 0.5 .

In the beryllium series, the electron that is ionized occupies the $2 \mathrm{~s}$ orbital. Since there are four electrons and each moving in an elliptical orbit, each may at any time interact differently with the other three electrons. We suppose that the screening of the fourth electron increases by a half to 2 and the other electron in the $2 \mathrm{~s}$ orbital experiences a 
TABLE 3: Comparison of Lamb shifts for one, and two-electron atomic ions (eV).

\begin{tabular}{|c|c|c|c|c|c|}
\hline \multirow{2}{*}{$Z$} & (2) & (3) & (4) & (5) & (6) \\
\hline & $\begin{array}{c}\text { One } \\
\text { electron } \\
\text { (this work) }\end{array}$ & $\begin{array}{c}\text { One electron } \\
\text { (Johnson and } \\
\text { Soff) }\end{array}$ & $\begin{array}{c}\text { Two electron } \\
\text { (this work) }\end{array}$ & $\begin{array}{c}\text { Two electron } \\
\text { (Drake) }\end{array}$ & $\begin{array}{c}\text { Two electron } \\
\text { (Midtdal et } \\
\text { al.) }\end{array}$ \\
\hline 1 & 0.00003 & 0.00003 & & & \\
\hline 2 & 0.0005 & 0.0004 & 0.0002 & 0.0002 & 0.0002 \\
\hline 3 & 0.0022 & 0.0020 & 0.0012 & 0.0011 & 0.0010 \\
\hline 4 & 0.0060 & 0.0056 & 0.0039 & 0.0037 & 0.0034 \\
\hline 5 & 0.0131 & 0.0126 & 0.0093 & 0.0091 & 0.0081 \\
\hline 6 & 0.0241 & 0.0243 & 0.0182 & 0.0186 & 0.0164 \\
\hline 7 & 0.0415 & 0.0422 & 0.0328 & 0.0336 & 0.0292 \\
\hline 8 & 0.0666 & 0.0678 & 0.0541 & 0.0557 & 0.0475 \\
\hline 9 & 0.1208 & 0.1028 & 0.0856 & 0.0864 & 0.0724 \\
\hline 10 & 0.1465 & 0.1489 & 0.1243 & 0.1275 & 0.1047 \\
\hline 11 & 0.2082 & 0.2079 & 0.1793 & 0.1806 & 0.1453 \\
\hline 12 & 0.2789 & 0.2817 & 0.2434 & 0.2476 & 0.1948 \\
\hline 13 & 0.3748 & 0.3720 & 0.3305 & 0.3303 & 0.2538 \\
\hline 14 & 0.4809 & 0.4808 & 0.4280 & 0.4306 & 0.3226 \\
\hline 15 & 0.6204 & 0.6101 & 0.5565 & 0.5504 & 0.4013 \\
\hline 16 & 0.7708 & 0.7620 & 0.6962 & 0.6916 & 0.4900 \\
\hline 17 & 0.9642 & 0.9383 & 0.8762 & 0.8562 & 0.5883 \\
\hline 18 & 1.2104 & 1.1414 & 1.1058 & 1.0461 & 0.6957 \\
\hline 19 & 1.4269 & 1.3724 & 1.3099 & 1.2634 & 0.8116 \\
\hline 20 & 1.6957 & 1.6346 & 1.5634 & 1.5101 & 0.9348 \\
\hline
\end{tabular}

TABLE 4: Screening constants.

\begin{tabular}{lccc}
\hline Isoelectronic series & $S$ & $S_{1}$ & $S_{2}$ \\
\hline Helium series & 0.5 & 0.0 & 0.5 \\
Lithium series & 1.5 & 0.5 & 0.625 \\
Beryllium series & 2.0 & 1.5 & 1.75 \\
Boron series & 3.0 & 2.0 & 2.25 \\
\hline
\end{tabular}

screening of between 1.5 and 2 and is 1.75 . After ionization of the fourth electron, the screening experienced by the third electron drops back to 1.5 .

For the boron system, the outermost electron occupies a new orbital and the screening increases by 1 to 3 . The screening of the fourth electron increases by 0.25 to 2.25 and after ionization the screening reduces back to 2 . There is no reason to expect screening constants to be complicated numbers, and the screening constants used in the calculations are listed in Table 4.

\section{Residual Electron Interaction}

The reduced mass calculation implicitly assumes that the electron and nucleus are point charges, but the nucleus and the electron have a finite size [22]. We assume that when there is more than one electron in the atom/ion, the finite size of the electron generates two opposite and competing kinds of residual electron-electron interactions. The first type is residual electron-electron repulsion because the electrons are not point charges (have a finite size) and are slightly closer to each other. This increases the electron-electron repulsion and reduces the energy required to ionize the electron. However, this reduction diminishes very rapidly with increasing number of protons as the electrons become much more tightly bound to the nucleus.

This residual electron-electron repulsion is directly proportional to the number of electron-electron interactions and an inverse function of proton-electron interactions. The number of electron-electron interactions $\mathrm{Q}_{\mathrm{I}}$, before ionization, is 1,3 , and 6 when there are two, three, or four electrons and so on. The reduction in energy resulting from this interaction is approximated to

$$
E_{r 1}=\left(\frac{1}{2} m_{0} v_{0}^{2}\right) Q_{I} \sqrt{\frac{\left(r_{e} / r_{B}\right)}{(Z-(I-1)) !}}
$$

$r_{e}$, the electron radius, is $e^{2} / 4 \pi \varepsilon_{0} m_{e} c^{2}, r_{\mathrm{B}}$ is the Bohr radius $\left(\varepsilon_{0} h^{2} / \pi m_{e} c^{2}\right)$, and $I$ is the number of electrons after ionization.

We assume that the second type of residual interaction, which is opposite to the first type and a competing type of electron-electron interaction, occurs when there are three or more electrons in the system before ionization. In a threeelectron system, two electrons occupy the 1 s orbital and one occupies the $2 \mathrm{~s}$ orbital. The finite size of the two electrons causes them to repel each other slightly more and tend to 
TABLE 5: Comparison of the ionization energies of one-electron atomic ions (eV).

\begin{tabular}{|c|c|c|c|c|c|}
\hline \multirow{2}{*}{$Z$} & (2) & (3) & $(4)$ & (5) & (6) \\
\hline & This paper & Johnson and Soff & Garcia and Mack & CRC & Diff $\%$ \\
\hline 1 & 13.5984 & 13.5984 & 13.5984 & 13.5984 & 0.0001 \\
\hline 2 & 54.4177 & 54.4178 & 54.4178 & 54.4178 & 0.0001 \\
\hline 3 & 122.4541 & 122.4543 & 122.4543 & 122.4543 & 0.0001 \\
\hline 4 & 217.7182 & 217.7185 & 217.7185 & 217.7187 & 0.0002 \\
\hline 5 & 340.2256 & 340.2260 & 340.2259 & 340.2258 & 0.0001 \\
\hline 6 & 489.9935 & 489.9931 & 489.9931 & 489.9933 & 0.0000 \\
\hline 7 & 667.0470 & 667.0460 & 667.0459 & 667.0460 & -0.0001 \\
\hline 8 & 871.4116 & 871.4098 & 871.4095 & 871.4101 & -0.0002 \\
\hline 9 & 1103.1187 & 1103.1173 & 1103.1170 & 1103.1176 & -0.0001 \\
\hline 10 & 1362.2039 & 1362.1990 & 1362.1987 & 1362.1995 & -0.0003 \\
\hline 11 & 1648.7060 & 1648.7019 & 1648.7015 & 1648.7020 & -0.0002 \\
\hline 12 & 1962.6733 & 1962.6634 & 1962.6629 & 1962.6650 & -0.0004 \\
\hline 13 & 2304.1485 & 2304.1397 & 2304.1391 & 2304.1410 & -0.0003 \\
\hline 14 & 2673.1951 & 2673.1772 & 2673.1768 & 2673.1820 & -0.0005 \\
\hline 15 & 3069.8581 & 3069.8411 & 3069.8411 & 3069.8420 & -0.0005 \\
\hline 16 & 3494.2184 & 3494.1874 & 3494.1884 & 3494.1892 & -0.0008 \\
\hline 17 & 3946.3218 & 3946.2904 & 3946.2931 & 3946.2960 & -0.0007 \\
\hline 18 & 4426.2335 & 4426.2222 & 4426.2273 & 4426.2296 & -0.0001 \\
\hline 19 & 4934.1039 & 4934.0476 & 4934.0574 & 4934.0460 & -0.0012 \\
\hline 20 & 5469.9500 & 5469.8608 & 5469.8776 & 5469.8640 & -0.0016 \\
\hline
\end{tabular}

TABLE 6: Components of the ionization energies of two-electron atomic ions (eV).

\begin{tabular}{|c|c|c|c|c|c|}
\hline \multirow{2}{*}{$Z$} & (2) & (3) & (4) & (5) & (6) \\
\hline & $E_{k}$ & $E_{l}$ & $E_{t}$ & $E_{r}$ & $I_{2}$ \\
\hline 1 & 3.3996 & 0.0000 & 2.5497 & 0.0993 & 0.7507 \\
\hline 2 & 30.6094 & 0.0002 & 5.9517 & 0.0702 & 24.5874 \\
\hline 3 & 85.0353 & 0.0012 & 9.3532 & 0.0405 & 75.6404 \\
\hline 4 & 166.6840 & 0.0039 & 12.7546 & 0.0203 & 153.9053 \\
\hline 5 & 275.5684 & 0.0093 & 16.1560 & 0.0091 & 259.3941 \\
\hline 6 & 411.7027 & 0.0182 & 19.5573 & 0.0037 & 392.1235 \\
\hline 7 & 575.1094 & 0.0328 & 22.9587 & 0.0014 & 552.1166 \\
\hline 8 & 765.8109 & 0.0541 & 26.3601 & 0.0005 & 739.3961 \\
\hline 9 & 983.8362 & 0.0856 & 29.7616 & 0.0002 & 953.9889 \\
\hline 10 & 1229.2125 & 0.1243 & 33.1630 & 0.0001 & 1195.9253 \\
\hline 11 & 1501.9819 & 0.1793 & 36.5644 & 0.0000 & 1465.2381 \\
\hline 12 & 1802.1778 & 02434 & 39.9658 & 0.0000 & 1761.9686 \\
\hline 13 & 2129.8508 & 0.3305 & 43.3673 & 0.0000 & 2086.1531 \\
\hline 14 & 2485.0427 & 0.4280 & 46.7686 & 0.0000 & 2437.8461 \\
\hline 15 & 2867.8127 & 0.5565 & 50.1701 & 0.0000 & 2817.0861 \\
\hline 16 & 3278.2110 & 0.6962 & 53.5715 & 0.0000 & 3223.9433 \\
\hline 17 & 3716.3056 & 0.8762 & 56.9729 & 0.0000 & 3658.4565 \\
\hline 18 & 4182.1621 & 1.1058 & 60.3744 & 0.0000 & 4120.6818 \\
\hline 19 & 4675.8384 & 1.3099 & 63.7758 & 0.0000 & 4610.7527 \\
\hline 20 & 5197.4218 & 1.5634 & 67.1772 & 0.0000 & 5128.6812 \\
\hline
\end{tabular}


TABLE 7: Comparison of ionization energies of two-electron atomic ions (eV).

\begin{tabular}{|c|c|c|c|c|c|}
\hline \multirow{2}{*}{$Z$} & (2) & (3) & (4) & (5) & (6) \\
\hline & This work & Drake & Midtdal et al. & $\mathrm{CRC}$ & Diff \% \\
\hline \multicolumn{6}{|l|}{1} \\
\hline 2 & 24.5874 & 24.5874 & 24.5874 & 24.5874 & 0.0001 \\
\hline 3 & 75.6404 & 75.6401 & 75.6402 & 75.6400 & -0.0005 \\
\hline 4 & 153.9053 & 153.8962 & 153.8965 & 153.8966 & -0.0057 \\
\hline 5 & 259.3941 & 259.3743 & 259.3753 & 259.3752 & -0.0073 \\
\hline 6 & 392.1235 & 392.0905 & 392.0926 & 392.0870 & -0.0093 \\
\hline 7 & 552.1166 & 552.0673 & 552.0715 & 552.0718 & -0.0081 \\
\hline 8 & 739.3961 & 739.3267 & 739.3344 & 739.2900 & -0.0144 \\
\hline 9 & 953.9889 & 953.8979 & 953.9107 & 953.9112 & -0.0081 \\
\hline 10 & 1195.9253 & 1195.8077 & 1195.8278 & 1195.8286 & -0.0081 \\
\hline 11 & 1465.2381 & 1465.0983 & 1465.1286 & 1465.1210 & -0.0080 \\
\hline 12 & 1761.9686 & 1761.8038 & 1761.8475 & 1761.8050 & -0.0093 \\
\hline 13 & 2086.1531 & 2085.9754 & 2086.0365 & 2085.9800 & -0.0083 \\
\hline 14 & 2437.8461 & 2437.6558 & 2437.7388 & 2437.6300 & -0.0089 \\
\hline 15 & 2817.0861 & 2816.9054 & 2817.0154 & 2816.9100 & -0.0063 \\
\hline 16 & 3223.9433 & 3223.7762 & 3223.9187 & 3223.7800 & -0.0051 \\
\hline 17 & 3658.4565 & 3658.3380 & 3658.5187 & 3658.5210 & 0.0018 \\
\hline 18 & 4120.6818 & 4120.6582 & 4120.8833 & 4120.8857 & 0.0049 \\
\hline 19 & 4610.7527 & 4610.7978 & 4611.0728 & 4610.8000 & 0.0010 \\
\hline 20 & 5128.6812 & 5128.8458 & 5129.1766 & 5128.8000 & 0.0023 \\
\hline
\end{tabular}

move further from each other. This asymmetric distribution of electrons causes the 2 s electron being screened slightly less from each of the $1 \mathrm{~s}$ electrons. The result is that it is attracted to the nucleus a bit more than expected and hence increases amount of energy required to remove it from the atom/ion. The small increase in energy is a function of the number of electron-electron interactions, there are because the more electron-electron interactions, the further they are apart and the bigger will be the reduction in screening of the nucleus from the ionizing electron. This effect also increases as the number of protons increases (i.e., attraction between the nucleus and outermost electron(s) increases), and for a system with three or more electrons it is approximately

$$
E_{r 2}=\frac{1}{n^{2}}\left(\frac{1}{2} m_{0} v_{0}^{2}(Z-S)^{2}\right) Q_{\mathrm{II}} \sqrt{\frac{\left(r_{e} / r_{B}\right)}{2^{2}}}
$$

$Q_{\mathrm{II}}$ is the number of electron interactions after ionization and the total residual electron interaction energy change is

$$
\begin{aligned}
E_{r}= & -\left(\frac{1}{2} m_{0} v_{0}^{2}\right) Q_{\mathrm{I}} \sqrt{\frac{\left(r_{e} / r_{B}\right)}{(Z-(I-1)) !}} \\
& +\frac{1}{n^{2}}\left(\frac{1}{2} m_{0} v_{0}^{2}(Z-S)^{2}\right) Q_{\mathrm{II}} \sqrt{\frac{\left(r_{e} / r_{B}\right)}{2^{2}}} .
\end{aligned}
$$

\section{Ionization Energy of One-Electron Atoms}

There is only one electron, $n$ is 1 , and the expression for calculating the ionization energy is

$$
\begin{aligned}
E=\mu & \frac{1}{2} m_{o} v^{2}+0.5\left(\frac{(1 / 4) m_{o} v^{4}}{c^{2}}+\frac{(3 / 16) m_{o} v^{6}}{c^{4}}\right) \\
& \left.-\frac{m_{e}}{\left(m_{e}+m_{p}\right)} \times\left[\left(\frac{\alpha}{2^{0.667}}\right)\left(\left(\frac{1}{2}\right) m_{o} v_{0}^{2}\right)(Z)^{3.2} A^{1 / 3}\right]\right) .
\end{aligned}
$$

Column 2 of Table 2 shows the nonrelativistic kinetic energy $\mu\left((1 / 2) m_{0} v^{2}\right)$, for the purpose of comparison, column 3 shows the relativistic correction calculated by (5), column 4 shows the relativistic correction calculated by (9), column 5 shows the Lamb shift correction, column 6 shows the ionization energy calculated by expression (6), and column 7 shows the ionization energy calculated by expression (16) (which is equivalent to (10)).

The one-electron ionization energies calculated by (16) are shown in column 2 of Table 5. Ionization energies computed by Johnson and Soff [7] and Garcia and Mack [5] and values published in the CRC Handbook are shown in columns 3,4 , and 5 , respectively. It is very clear that (9) is a much more appropriate expression than (5) to calculate the relativistic correction. Ionization energies calculated by expression (16) show exceptional agreement with values computed by complex formulas and with the latest accepted values. Compared to ionization energies published in the CRC Handbook, values calculated by (16) agree to $99.999 \%$ or differ by less than $0.001 \%$ (or $<10$ parts per million) in 18 out 
of the 20 values as shown in column 6 of Table 5, the biggest absolute difference is $0.0860 \mathrm{eV}$ from a calculated value of $5469.95 \mathrm{eV}$.

\section{Ionization Energy of Two-Electron Atoms}

The ionization energy of a two-electron system is $\left(E_{k}-E_{l}-\right.$ $E_{t} \pm E_{r}$ ), where:

$$
\begin{gathered}
E_{l}=\mu\left(\frac{m_{e}}{\left(m_{e}+m_{p}\right)} \times\left[\left(\frac{\alpha}{2^{2 / 3}}\right)\left(\left(\frac{1}{2}\right) m_{0} v_{0}^{2}\right)(Z-0.5)^{3.2} A^{1 / 3}\right]\right) \\
E_{t}=0.25 \mu\left(\frac{1}{2} m_{0}\left(v_{0}(Z)\right)^{2}-\frac{1}{2} m_{0}\left(v_{0}(Z-0.5)\right)^{2}\right) \\
E_{r}=\left(-\left(\frac{1}{2} m_{0} v_{0}^{2}\right) Q_{\mathrm{I}} \sqrt{\frac{\left(r_{e} / r_{B}\right)}{(Z) !}}\right) .
\end{gathered}
$$

Since, for simplicity, we have not applied a relativistic correction to $E_{t}$, we have made a crude approximation of reducing the relativistic correction in $E_{k}$ by $5 \%$ to 0.45 (rather than 0.5$)$ and where $v$ is $\left(v_{0}(Z-0.5)\right)$, so it is

$$
E_{k}=\mu\left(\frac{1}{2} m_{0} v^{2}+0.45\left(\frac{(1 / 4) m_{0} v^{4}}{c^{2}}+\frac{(3 / 16) m_{0} v^{6}}{c^{4}}\right)\right)
$$

Columns 2, 3, 4, 5, and 6 of Table 6 show $E_{k}, E_{l}, E_{t}, E_{r}$ separately and the calculated ionization energies of twoelectron atoms, respectively. Table 7 is a comparison of the ionization energies calculated in this paper and those computed by Drake [8], Midtdal, and Aashamar [6] and values published in the CRC Handbook. As shown in the table, there is very good agreement with the other computed results. When compared to values published in the CRC Handbook, all values except one (atomic number 8 ) agree to better than 99.99\% (or difference of less than $0.01 \%$ as shown in column 6 ), and the largest absolute difference is $0.2161 \mathrm{eV}$ from a calculated value of $2437.8641 \mathrm{eV}$.

\section{Ionization Energy of Three, Four, and Five Electron Atoms}

The ionization energy of a three- to-five electron system is

$$
\left(E_{k}-E_{l}-E_{t} \pm E_{r}\right)
$$

where

$$
\begin{gathered}
E_{k}=0.25 \mu\left(\frac{1}{2} m_{0} v^{2}+0.5\left(\frac{(1 / 4) m_{0} v^{4}}{c^{2}}+\frac{(3 / 16) m_{0} v^{6}}{c^{4}}\right)\right), \\
E_{l}=0.25 \mu\left(\frac{m_{e}}{m_{e}+m_{p}}\left(\frac{\alpha}{2^{2 / 3}}\right)\left(\frac{1}{2} m_{0} v_{0}^{2}\right)(Z-S)^{3.2} A^{1 / 3}\right), \\
E_{t}=\mu\left(\frac{1}{2} m_{0}\left(v_{0}\left(Z-S_{1}\right)\right)^{2}-\frac{1}{2} m_{0}\left(v_{0}\left(Z-S_{2}\right)\right)^{2}\right), \\
E_{r}=\left(-\left(\frac{1}{2} m_{0} v_{0}^{2}\right) Q_{\mathrm{I}} \sqrt{\frac{\left(r_{e} / r_{B}\right)}{(Z-(I-1)) !}}\right. \\
\left.+\frac{1}{n^{2}}\left(\frac{1}{2} m_{0} v_{0}^{2}(Z-S)^{2}\right) Q_{\mathrm{II}} \sqrt{\frac{\left(r_{e} / r_{B}\right)}{2^{2}}}\right) .
\end{gathered}
$$

The screening constants used are shown in Table 4, and $Q_{\mathrm{I}}$, the number of electron-electron interactions before ionization, is 3,6 , and 10, respectively, for three-, four, and five-electron systems. Columns 2, 3, and 4 of Table 8 list the calculated ionization energies, literature values, and the percentage differences of three-electron isoelectronic systems. The calculated ionization energies of four- and fiveelectron isoelectronic sequences are shown in columns 2 and 5 ; the corresponding literature values in columns 3 and 6 and their differences are shown in columns 4 and 7, respectively, in Table 9. The agreement with literature values is $99 \%$ or better in all cases (99.9\% or better for a third of all values).

\section{First Electron Affinities}

First electron affinities calculated by our expression and recommended values are listed in Table 10. Our calculated value of the first electron affinity of helium differs from the generally accepted value by less than $0.5 \%$ which is an excellent agreement. There is also fairly good agreement between our other calculated values and recommended values.

\section{Discussion}

Johnson and Soff [7] used an approximate empirical formula as part of the set of expressions to calculate the finite nuclear size correction. Drake [8] reported that uncertainties of calculated Lamb shifts in some cases can be up to $10 \%$. Our expression (19) is fairly simple and can be calculated quite easily as compared to the complex equations, which required computer routines to compute.

We have only used the size of the (one-electron) hydrogen atom in the Lamb shift equation for one to five electrons. It is fairly remarkable that in the majority of cases agreement between our calculated values and the values computed by Johnson and Soff [7] and Drake [8] is 90\% or better. The size of hydride ion (negative hydrogen ion) is different from the hydrogen atom, and obviously the sizes of the negative helium, lithium, and beryllium ions are as well. We have not applied any corrections to negative ions for the twoto five-electron systems, because the size of the hydride ion 
TABLE 8: Ionization energies of three electron atom/atomic ions $(\mathrm{eV})$.

\begin{tabular}{|c|c|c|c|c|c|}
\hline \multirow{2}{*}{$Z$} & (2) & (3) & (4) & (5) & (6) \\
\hline & This work & CRC & Diff $\%$ & 1 & 0.999455717 \\
\hline 2 & & & & 2 & 0.999862962 \\
\hline 3 & 5.3975 & 5.3917 & -0.1075 & 3 & 0.999921837 \\
\hline 4 & 18.2921 & 18.2111 & -0.4445 & 4 & 0.999939160 \\
\hline 5 & 37.9891 & 37.9306 & -0.1540 & 5 & 0.999950207 \\
\hline 6 & 64.4924 & 64.4939 & 0.0024 & 6 & 0.999954325 \\
\hline 7 & 97.8161 & 97.8902 & 0.0757 & 7 & 0.999960870 \\
\hline 8 & 137.9734 & 138.1197 & 0.1059 & 8 & 0.999965753 \\
\hline 9 & 184.9757 & 185.1860 & 0.1136 & 9 & 0.999971178 \\
\hline 10 & 238.8325 & 239.0989 & 0.1114 & 10 & 0.999972620 \\
\hline 11 & 299.5532 & 299.8640 & 0.1036 & 11 & 0.999972620 \\
\hline 12 & 367.1481 & 367.5000 & 0.0957 & 12 & 0.999977196 \\
\hline 13 & 441.6271 & 442.0000 & 0.0844 & 13 & 0.999979768 \\
\hline 14 & 523.0034 & 523.4200 & 0.0796 & 14 & 0.999980467 \\
\hline 15 & 611.2877 & 611.7400 & 0.0739 & 15 & 0.999982367 \\
\hline 16 & 706.4970 & 707.0100 & 0.0726 & 16 & 0.999982925 \\
\hline 17 & 808.6426 & 809.4000 & 0.0936 & 17 & 0.999984398 \\
\hline 18 & 917.7394 & 918.0300 & 0.0317 & 18 & 0.999986358 \\
\hline 19 & 1033.8184 & 1033.4000 & -0.0405 & 19 & 0.999986014 \\
\hline 20 & 1156.8864 & 1157.8000 & 0.0789 & 20 & 0.999986372 \\
\hline
\end{tabular}

TABLE 9: Ionization energies of four- and five-electron atom/atomic ions (eV).

\begin{tabular}{|c|c|c|c|c|c|c|}
\hline \multirow{2}{*}{$Z$} & (2) & (3) & (4) & $(5)$ & (6) & $(7)$ \\
\hline & This work (four electrons) & CRC & Diff \% & This work (five electrons) & $\mathrm{CRC}$ & Diff \% \\
\hline \multicolumn{7}{|l|}{3} \\
\hline 4 & 9.2942 & 9.3227 & 0.3058 & & & \\
\hline 5 & 24.9666 & 25.1548 & 0.7482 & 8.3121 & 8.2980 & -0.1695 \\
\hline 6 & 47.4641 & 47.8878 & 0.8849 & 24.2893 & 24.3833 & 0.3855 \\
\hline 7 & 76.7925 & 77.4735 & 0.8791 & 47.1286 & 47.4492 & 0.6758 \\
\hline 8 & 112.9756 & 113.8990 & 0.8107 & 76.8369 & 77.4135 & 0.7449 \\
\hline 9 & 156.0359 & 157.1651 & 0.7185 & 113.4510 & 114.2428 & 0.6931 \\
\hline 10 & 205.9899 & 207.2759 & 0.6204 & 157.0043 & 157.9300 & 0.5861 \\
\hline 11 & 262.8503 & 264.2500 & 0.5297 & 207.5202 & 208.5000 & 0.4699 \\
\hline 12 & 326.6286 & 328.0600 & 0.4363 & 265.0149 & 265.9600 & 0.3553 \\
\hline 13 & 397.3348 & 398.7500 & 0.3549 & 329.5004 & 330.1300 & 0.1907 \\
\hline 14 & 474.9817 & 476.3600 & 0.2893 & 400.9890 & 401.3700 & 0.0949 \\
\hline 15 & 559.5797 & 560.8000 & 0.2176 & 479.4916 & 479.4600 & -0.0065 \\
\hline 16 & 651.1450 & 652.2000 & 0.1618 & 565.0208 & 564.4400 & -0.1029 \\
\hline 17 & 749.6888 & 749.7600 & 0.0095 & 657.5892 & 656.7100 & -0.1339 \\
\hline 18 & 855.2253 & 854.7700 & -0.0533 & 757.2096 & 755.7400 & -0.1945 \\
\hline 19 & 967.7837 & 968.0000 & 0.0223 & 863.9076 & 861.1000 & -0.3261 \\
\hline 20 & 1087.3713 & 1087.0000 & -0.0342 & 977.6915 & 974.0000 & -0.3790 \\
\hline
\end{tabular}

[23] is contentious and there is no accurate measurement of the size of the other negative ions. There are no reliable Lamb shift calculations for three- to five-electron systems, so a comparison with our results is not possible. Even though our assumptions and approximations are simple and straight forward, our results show excellent agreement with ionization energies published in the CRC Handbook as well as with
Johnson and Soff [7] and Garcia and Mack [5] for oneelectron and Midtdal and Aashamar [6] and Drake [8] for two-electron ions.

We have found that, as shown by up-to-date publications [24], Moore is still quoted as a major source of experimental information. For some isoelectronic sequences, only the first five or six members are reliably measured and there may 
TABLE 10: First electron affinities (eV).

\begin{tabular}{lcccc}
\hline$(1)$ & $(2)$ & $(3)$ & $(4)$ & $(5)$ \\
Element & Hydrogen & Helium & Lithium & Beryllium \\
\hline Calculated & 0.7507 & -0.6667 & 0.5045 & -0.4824 \\
Recommended $^{*}$ & 0.7542 & $<0$ & 0.6180 & $<0$ \\
\hline
\end{tabular}

Recommended values are from [16].

be many members somewhere along the sequence without any reliably measured ionization energies, and in these cases values are obtained by semiempirical interpolation or extrapolation formulas [25]. Recent papers [26, 27] also quoted ionization energies of some members of many isoelectronic sequences estimated by Lotz [28] in 1967. We have recently demonstrated $[29,30]$ that ionization energies and ionization pathways of some elements or sequences are fairly complicated. Hence, there is scope to update the measurements and calculations of ionization energies of isoelectronic sequences.

Although some of our hypotheses may be controversial and there is no proof that our interpretation of the relativistic correction for mass change is correct, there is no doubt that they form part of expressions that produce very precise values of the ionization energies of one- to five-electron isoelectronic sequences. This is good evidence that both expression (19) or our alternative potential energy approach can be developed to predict, with good accuracy, ionization energies of multi-electron atomic ions that have not been reliably measured.

\section{References}

[1] P. A. M. Dirac, Principles of Quantum Mechanics, Chapter 5, Oxford University Press, Oxford, UK, 1930.

[2] W. E. Lamb and R. C. Retherford, "Fine structure of the hydrogen atom by a microwave method," Physical Review, vol. 72, no. 3, pp. 241-243, 1947.

[3] W. E. Lamb and R. C. Retherford, "Fine structure of the hydrogen atom. Part I," Physical Review, vol. 79, no. 4, pp. 549-572, 1950

[4] W. E. Lamb and R. C. Retherford, "Fine structure of the hydrogen atom. Part II," Physical Review, vol. 81, no. 2, pp. 222-232, 1951.

[5] J. D. Garcia and J. E. Mack, "Energy level and line tables for one-electron atomic spectra," Journal of the Optical Society of America, vol. 55, no. 6, pp. 654-676, 1965.

[6] J. Midtdal and K. Aashamar, "Perturbation theory expansions through 20th order of mass polarization correction, relativistic effects and Lamb shift of the two-electron system (1s) ${ }^{2}$," Physica Norvegica, vol. 2, pp. 99-108, 1967.

[7] W. R. Johnson and G. Soff, "The lamb shift in hydrogen-like atoms, $1 \leq \mathrm{Z} \leq 110$," Atomic Data and Nuclear Data Tables, vol. 33, no. 3, pp. 405-446, 1985.

[8] G. W. Drake, "Theoretical energies for the $n=1$ and 2 states of the helium isoelectronic sequence up to $Z=100$," Canadian Journal of Physics, vol. 66, pp. 586-611, 1988.

[9] P. F. Lang and B. C. Smith, "Ionisation potentials of one-electron atoms," Inorganic and Nuclear Chemistry Letters, vol. 17, pp. 27-. 29, 1981.
[10] A. M. E. Sabir, P. F. Lang, and B. C. Smith, "Ionisation energies of two-electron atoms," Journal of the Chemical Society, vol. 80, pp. 1089-1091, 1984.

[11] P. F. Lang and B. C. Smith, "A simple formula to calculate the ionization energies of two-, three-, and four-electron atomic ions," Naturwissenschaften, vol. 97, no. 7, pp. 689-696, 2010.

[12] C. E. Moore, Atomic Energy Levels, vol. 1, US Department of Commerce, Washington, DC, USA, 1949.

[13] C. E. Moore, Atomic Energy Levels, vol. 2, US Department of Commerce, Washington, DC, USA, 1952.

[14] C. E. Moore, Atomic Energy Levels, vol. 3, US Department of Commerce, Washington, DC, USA, 1958.

[15] C. E. Moore, Ionization Potentials and Ionization Limits Derived From the Analysis of Optical Spectra, NSRDS-NBS 34, US Department of Commerce, Washington, DC, USA, 1970.

[16] D. R. Lide, CRC Handbook of Chemistry and Physics, CRC, Boca Raton, Fla, USA, 89th edition, 2009.

[17] T. Andersen, H. K. Haugen, and H. J. Hotop, "Binding energies in atomic negative ions: III," Journal of Physical and Chemical Reference Data, vol. 28, no. 1511, 1527 pages, 1999.

[18] E. U. Condon and H. Odabasi, Atomic Structure, Chapter 1, CUP, New York, NY, USA, 1980.

[19] A. Sommerfeld, Atomic Structure and Spectral Lines, Chapter 2, Methuen, London, UK, 1934.

[20] W. G. V. Rosser, Relativity and High Energy Physics, Chapter 2, Methuen, London, UK, 1969.

[21] I. R. Williams and M. W. Williams, Basic Nuclear Physics, Chapter 2, Newnes, London, UK, 1962.

[22] M. Born, Atomic Physics, Chapter 3, Blackie, London, UK, 8th edition, 1969.

[23] P. F. Lang and B. C. Smith, "Ionic radii for group 1 and group 2 halide, hydride, fluoride, oxide, sulfide, selenide and telluride crystals," Dalton Transactions, vol. 39, no. 33, pp. 7786-7791, 2010.

[24] J. E. Sansonetti and W. C. Martin, "Handbook of basic atomic spectroscopic data," Journal of Physical and Chemical Reference Data, vol. 34, pp. 1559-1992, 2005.

[25] J. E. Sansonetti, "Wavelengths, transition probabilities, and energy levels for the spectra of rubidium (RbI through RbVII)," Journal of Physical and Chemical Reference Data, vol. 35, pp. 301-421, 2006.

[26] J. Sugar and A. Musgrove, "Energy levels of Zinc, Zn I through Zn," Journal of Physical and Chemical Reference Data, vol. 24, pp. 1803-1872, 1995.

[27] T. Shirai, J. Sugar, and A. Musgrove, "Spectral data for highly ionized atoms: $\mathrm{Ti}, \mathrm{V}, \mathrm{Cr}, \mathrm{MN}, \mathrm{Fe}, \mathrm{Co}, \mathrm{Ni}, \mathrm{Cu}, \mathrm{Kr}$, and $\mathrm{Mo}$," Journal of Physical and Chemical Reference Data, Monograph No. 8, 632 pages, 2000.

[28] W. Lotz, "Ionization potentials of atoms and ions from hydrogen to Zinc," Journal of the Optical Society of America, vol. 57, pp. 873-880, 1967.

[29] P. F. Lang and B. C. Smith, "Ionization energies of atoms and atomic ions," Journal of Chemical Education, vol. 80, no. 8, pp. 938-946, 2003.

[30] P. F. Lang and B. C. Smith, "Ionization energies of lanthanides," Journal of Chemical Education, vol. 87, no. 8, pp. 875-881, 2010. 

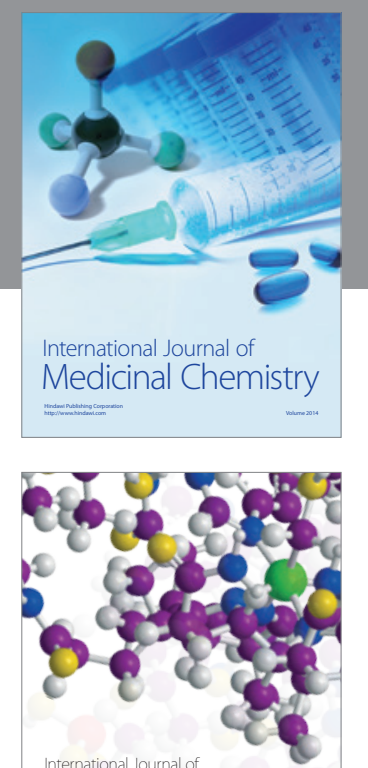

\section{Carbohydrate} Chemistry

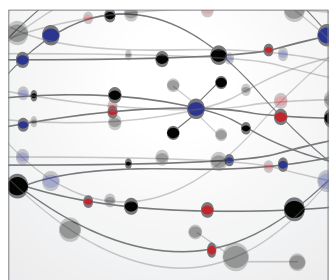

The Scientific World Journal
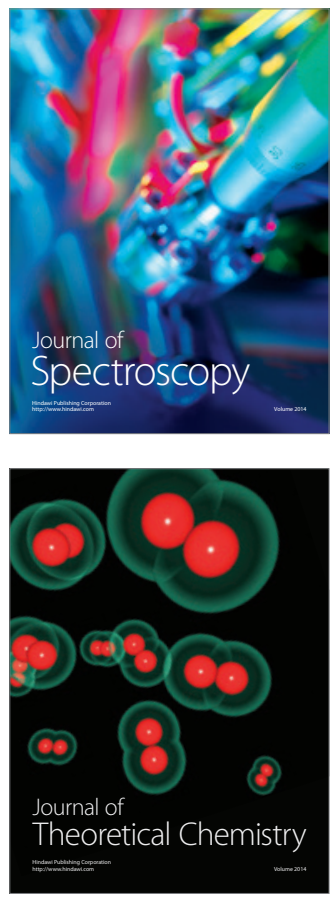
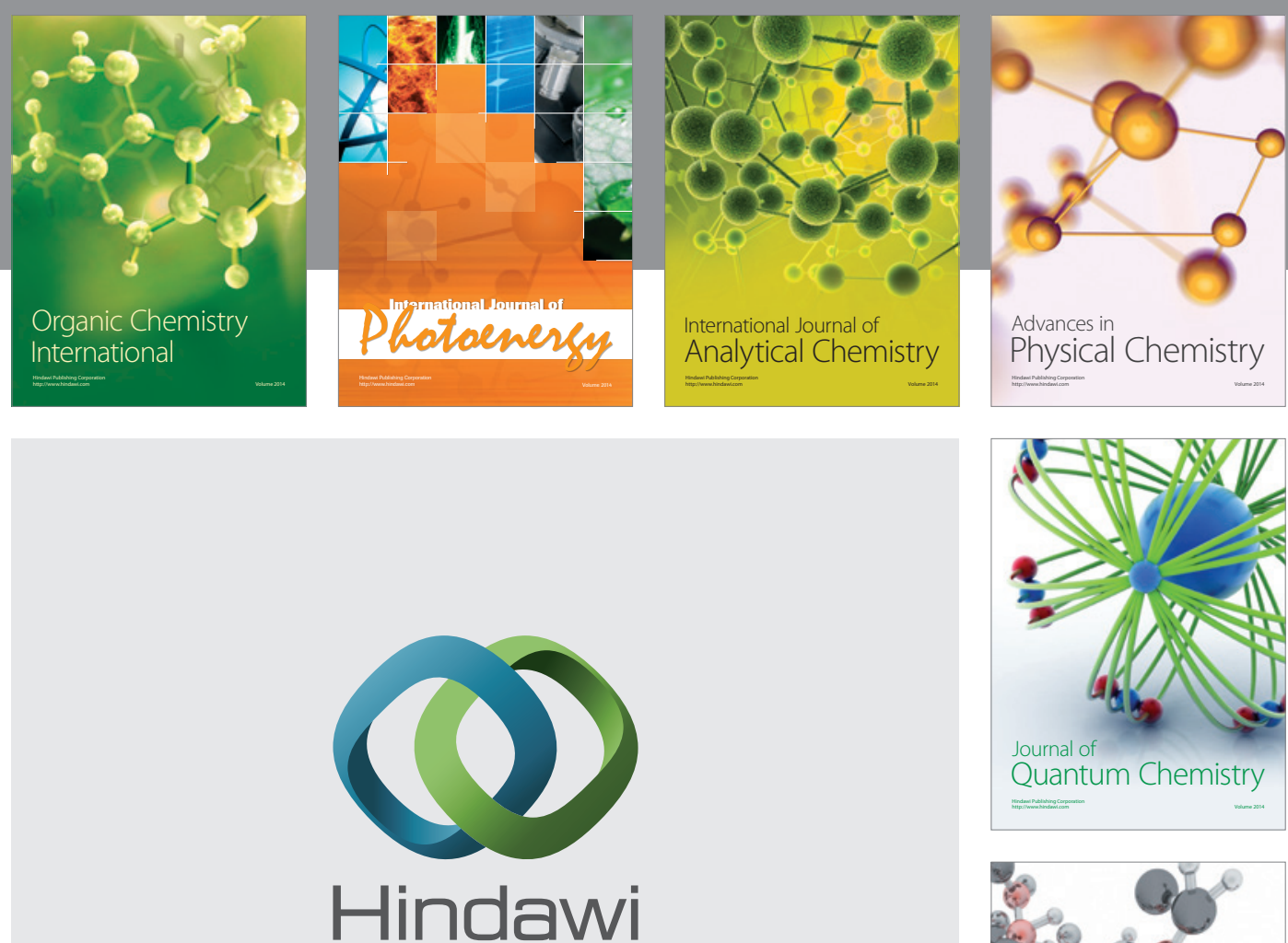

Submit your manuscripts at

http://www.hindawi.com

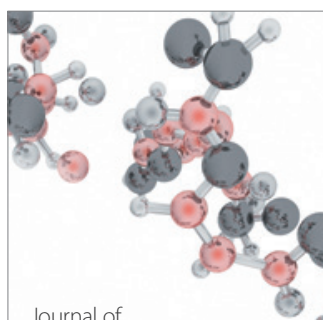

Analytical Methods

in Chemistry

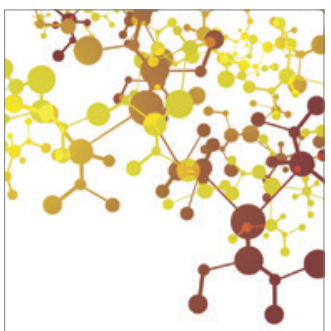

Journal of

Applied Chemistry

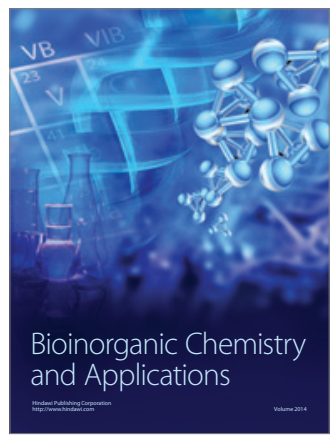

Inorganic Chemistry
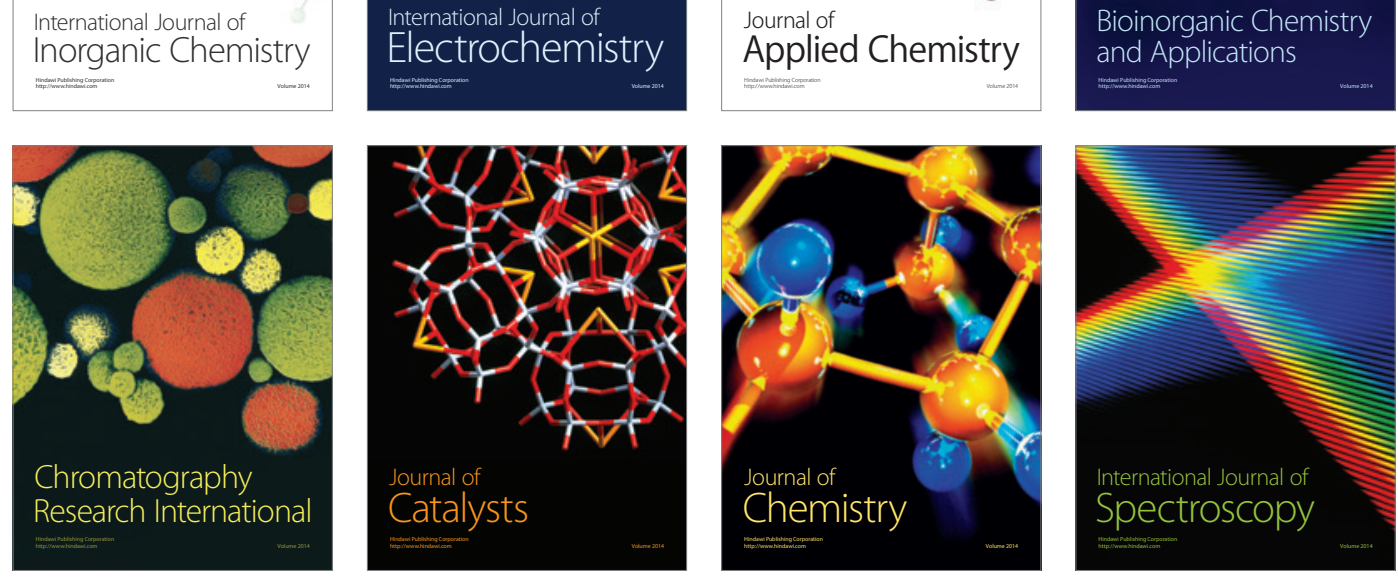